\title{
Do prostate cancer-related mobile phone apps have a role in contemporary prostate cancer management? A systematic review by EAU young academic urologists (YAU) urotechnology group
}

\author{
Enakshee Jamnadass ${ }^{1} \cdot$ Bhavan Prasad Rai $^{2} \cdot$ Domenico Veneziano $^{3} \cdot$ Theodoros Tokas $^{6} \cdot$ Juan Gomez Rivas $^{4}$. \\ Giovanni Cacciamani ${ }^{5}$. Bhaskar Somani ${ }^{1}$
}

Received: 14 November 2019 / Accepted: 31 March 2020 / Published online: 22 April 2020

(c) The Author(s) 2020

\begin{abstract}
Aims and objectives To review the available literature regarding the use of prostate cancer-related mobile phone applications (PCA).

Materials and methods The search was for English language articles between inceptions of databases to June 2019. Medline, EMBASE, Cochrane Library, CINAHL and Web of Science were searched. Full-text articles were reviewed, and the following data were extracted to aid with app analysis: name of application, developer, platform (Apple App Store or Google Play Store) and factors assessed by the article.

Results The search yielded 1825 results of which 13 studies were included in the final review. 44 PCAs were identified from the data collected of which 59\% of the PCAs had an educational focus. 11 apps were inactive and 5 weren't updated within the last year. Five studies focused on the development and testing of apps (MyHealthAvatar, CPC, Rotterdam, Interaktor, NED). Two studies evaluated the readability of PCAs. Most PCAs had a reading level greater than that of the average patient. Two studies evaluated the quality and accuracy of apps. Majority of PCAs were accurate with a wide range of information. The study reported most PCAs to have deficient or insufficient scores for data protection. Two studies evaluated the accuracy of Rotterdam, CORAL and CPC risk calculators. Rotterdam was the best performer.

Conclusions PCAs are currently in its infancy and do require further development before widespread integration into existing clinical practise. There are concerns with data protection, high readability standards and lack of information update in current PCAs. If developed appropriately with responsible governance, they do have the potential to play important roles in modern-day prostate cancer management
\end{abstract}

Keywords Prostate cancer $\cdot$ Mobile phone applications $\cdot$ Social media

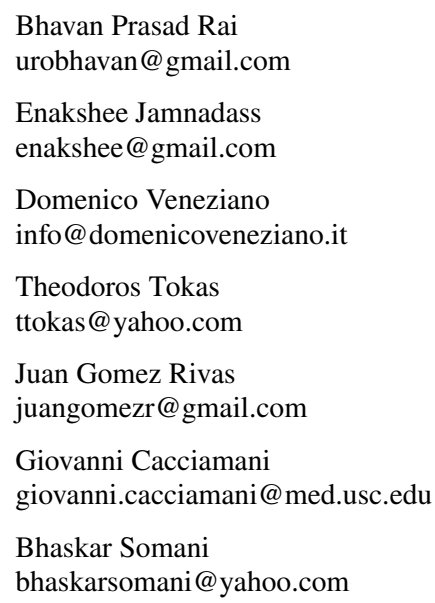

1 University Hospital Southampton NHS Trust, Southampton, UK

2 Freeman Hospital, Newcastle, UK

3 Department of Urology and Renal Transplantation, Bianchi-Melacrino-Morelli Hospital, Reggio Calabria, Italy

4 Department of Urology, Hospital Universitario La Paz, Madrid, Spain

5 Department of Urology, USC Urology Institute, University of Southern California, Los Angeles, CA, USA

6 Tirol Kliniken, Milser Strasse 10, Hall in Tirol, Austria 


\section{Introduction}

Information Communication Technology (ICT) is an integral part of modern-day healthcare delivery in domains such as education, research, operational efficiency and data management [1]. In prostate cancer with changing diagnostic and therapeutic paradigms, there is likely to be reliance on technology for the delivery of cost-effective, high-quality cancer care [2]. Mobile phone application (apps) is software with specific, limited function, which is designed for use on a mobile device [3]. It has been suggested mobile phone apps have the potential to increase patient awareness, be adjuncts to traditional clinical evaluation strategies and can also facilitate research development and delivery [4]. The two most popular platforms, from which users can download apps, are the Google Play Store, and the Apple App store. Over 2 million apps are available on these platforms [5]. There are over 5.5 billion smartphone users worldwide [6], and it is estimated that the average user spends over $3.5 \mathrm{~h}$ on their mobile device every day [7]. Furthermore, users spend $89 \%$ of their media time on mobile apps [8]. In the United Kingdom, a reported $75 \%$ of people go online for health information. Additionally, $70 \%$ of patients aged over 50 want to use digital healthcare services [9]. The market for healthcare-related apps is growing, and it is suggested that around 200 healthcare apps are added daily [9].

Prostate cancer is the most common cancer in males, in the UK (second most common in men, worldwide) and, according to the American Cancer Society, 1 in 9 men will be diagnosed with prostate cancer during their lifetime [10, 11]. Furthermore, the incidence of prostate cancer is increasing and is projected to rise in the UK by $12 \%$ between 2014 and 2035 [10]. Given the significance of prostate cancer worldwide, and the increasing usage of healthcare apps within patient populations, we aim to systematically review the available literature regarding the availability and usage of prostate cancer-related mobile phone apps (PCA). We also look at the type of app, its content, rating and their real-world application.

\section{Materials and methods}

\section{Selection criteria}

This review included studies that explored and evaluated various aspects of PCAs, as well as their current and potential applications in the screening, prevention or management of prostate cancer.

\section{Inclusion and exclusion criteria}

Inclusion criteria:

I. English-language oncological papers with a focus on prostate cancer.

II. Studies reporting on mobile phone apps for prostate cancer.

Exclusion criteria:

I. Literature reviews, grey literature, editorials, letters, and other 'comment' pieces.

II. Studies on prostate cancer not related to apps.

III. Studies relating to apps which are unpublished or unreleased.

\section{Search strategy}

This systematic review of world literature was performed in the Cochrane style and in accordance with the Preferred Reporting Items for Systematic Reviews and Meta-Analyses (PRISMA) checklist (Fig. 1) [10,11]. The search was for English language articles between inceptions of databases to June 2019, with the final search being conducted on 17/06/2019. Medline, EMBASE, Cochrane Library, CINAHL, and Web of Science were the databases searched. The search terms used were 'prostate', 'cancer', 'prostate cancer', 'PSA', 'prostate specific antigen', 'prevention', 'adenocarcinoma', 'prostatic intraepithelial neoplasia', 'social media', 'phone app', 'apps', 'search engine', 'online', 'web-based', 'ehealth', 'mhealth', 'user-generated content', 'mobile health', 'smartphone', 'mobile phone', 'personal digital assistant', 'google play', 'android', 'apple' and 'iOS'. Medical Subject Heading (MeSH) phrases included ("Prostatic Neoplasms"[MeSH]) AND "Mobile Applications" [MeSH]); ("Prostate", [MeSH]) AND "Smartphone application', [MeSH]); ("Prostate cancer',[MeSH]) AND “'Social media', [MeSH]) AND “'Mobile health', [MeSH]) aq. Boolean operators (AND, OR) were used to refine the search. Two reviewers (EJ and BS) identified all studies and those that appeared to fit the inclusion criteria were included for a full review. Papers evaluating a 'general' cancer app, that specifically mentioned prostate cancer patients within the article, were included. However, papers solely evaluating apps that did not have a cancer focus (e.g. pedometers or fitness trackers, without a cancer-related component) were excluded. Each reviewer independently selected studies for inclusion in the review and discrepancies were resolved by mutual consensus. A literature search had been run on each database. Any duplicates were excluded. At initial screening articles were excluded by title screening. The abstracts of 
Fig. 1 PRISMA

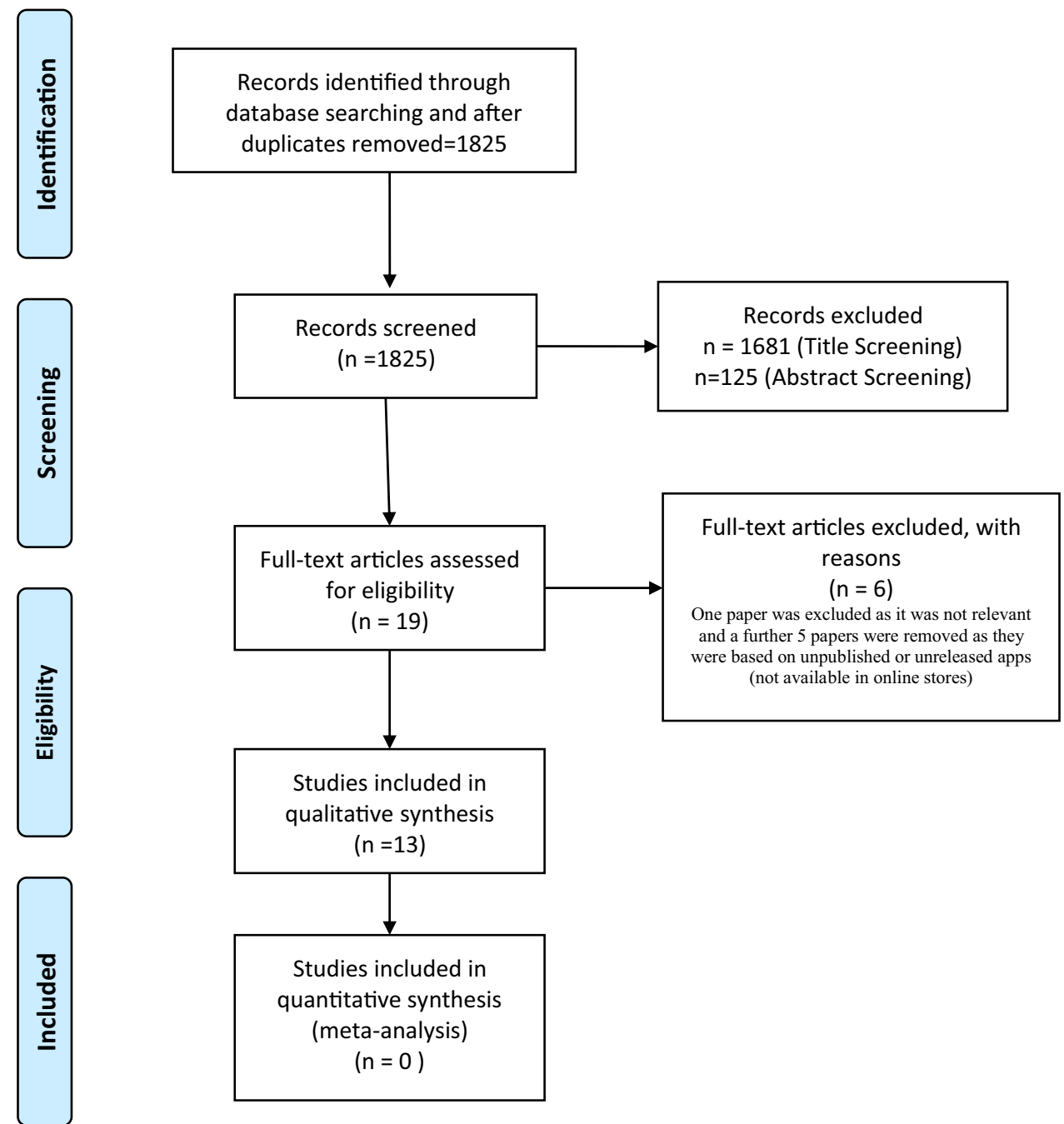

the remaining articles were further screened and excluded if considered unsuitable for the review. Full text of the remaining literature was then reviewed. After a complete evaluation of the full articles, articles were excluded if deemed unsuitable. The remaining studies were included in the review for a narrative synthesis. The following information was extracted and organised using a spreadsheet (to perform further analysis): the year of publication, journal, number of applications assessed, type of apps and assessment criteria used on the included applications. References for these studies were collected using EndNote Web, and citations were either imported directly or manually entered. The outcomes of individuals studies will be presented in narrative fashions with emphasis on App development, App Readability, Quality and Accuracy of Apps, App Usage, and Risk Calculators.

\section{Review of mobile phone applications (apps)}

The full-text articles were also assessed in detail for the apps and data was extracted for the following information (where available): name of application, developer, platform (Google Play Store or Apple App Store), and factors assessed by article. One of the authors (EJ) gathered further information on these apps which had been named by studies using the online stores (namely Google Play and App Store). This data included: the app's cost, star rating [1-5], number of reviews, date of the last update, and the advertised content of the app (found in the app's description). All information gained from both the online stores and from the literature was then compiled into the same spreadsheet for analysis. Data were collated using Microsoft Excel (version 12.2.4). 


\section{Results}

\section{Study selection}

The initial literature search yielded 1825 results; 449 from Medline, 539 from EMBASE, 159 from CINAHL, 188 from Cochrane Library, and 490 from Web of Science. One thousand seven hundred and fifty-two articles were screened after removal of duplicates. 1608 articles were removed after title screening. 125 articles were removed after the abstract screening. Full texts of 19 studies were assessed. Of these, one paper was excluded as it was not relevant and a further five papers were removed as they were based on unpublished or unreleased apps (not available in online stores). This left 13 papers for inclusion in our final review [12-24] (Fig. 1).

\section{Description of studies}

The papers included in this review focused on a variety of factors in assessing available apps including readability, quality and accuracy, usage, and app development. A comprehensive summary of the individual studies has been presented in Table 1. The authors have highlighted the outcomes of individual studies under the headings:

1. Studies related to app development.

2. Studies related to readability.

3. Studies related to 'quality and accuracy' of apps.

4. Studies related to app usage.

5. Studies related to risk calculators.

\section{Studies related to 'app development'}

Four studies focused on the development and testing of apps [18, 20, 21, 23]. Zhang et al. [23] presented the MyHealthAvatar app. This was a European Commission funded research project for patients with prostate and breast cancer. The app is available on desktop, tablet and smartphone. The app encourages patient self-management of their disease. It contains lifestyle and activity tracking. It has prostate and breast cancer questionnaires to monitor progress after treatment. It also provides advice on pelvic floor exercises for patients who have had a radical prostatectomy $[23,25]$. The app includes International Index of Erectile Function-5 (IIEF-5) and the International Prostate Symptom Score (I-PSS) questionnaires and resources from the Prostate Cancer UK and NHS, UK. They also tested user experience and outlined the early developmental flaws. Data from this app can support research activity.
Røder et al. [21, 26] developed and validated the CPC Risk Calculator which estimates the risk of biochemical recurrence following a radical prostatectomy. The authors used preoperative PSA, pT stage, prostatectomy Gleason score, and surgical margin $(R)$ status to develop the nomogram. The data was calculated from 2167 men who underwent a radical prostatectomy at the Copenhagen Prostate Cancer Center, Rigshospitalet, Denmark. The nomogram was externally validated using a cohort of 2237 men who underwent a radical prostatectomy at the Stanford University, California, USA in the same time period. The authors reported high accuracy and discrimination on external validation. The accuracy of model declined after 7 years due to limited follow-up in the 2 cohorts.

Pereira-Azevedo et al., evaluated their app, the Rotterdam Prostate Cancer Risk Calculator, via usability testing [20, 27]. Rotterdam Prostate Cancer Risk Calculator is developed using algorithms from Rotterdam arm of the European Randomized Study of Screening for Prostate Cancer (ERSPC) study. The calculator uses PSA level, previous negative prostate biopsy, digital rectal examination (DRE) findings, prostate volume measurement, transrectal ultrasonography findings, MRI results, and Prostate Health Index to estimate overall and significant prostate cancer risk. The app was scored by participants, on usefulness, quality of information, and quality of interface scoring highly (gaining $\geq 87 \%$ ) in all categories.

Langius-Eklöf et al. [18] tested their app, Interaktor, on prostate cancer patients undergoing radiotherapy, to determine its potential to ease symptom burden. They found high adherence to symptom reporting and realised a novel use for the app for clinicians, to determine the most commonly reported clinical side effects of their patients.

All four papers found that users benefited from, or had a neutral experience when using their apps, and authors explored potential possibilities for these apps to benefit healthcare professionals as well as patients (who the apps were designed for) [20, 22-25].

Pham et al., reported on a trial design to evaluate the acceptability of NED (No Evident disease) by patients, caregivers and clinicians. NED is a prostate cancer app developed to support prostate cancer survivorship programmes [24].

\section{Studies related to 'app readability'}

Kim et al. [17] and Owen et al. [19] tested the readability of cancer apps that are currently available online. Both papers used readability assessment tools to determine the gradelevel readability of each app. Kim et al. evaluated readability of PCAs along with four other cancer apps available on 


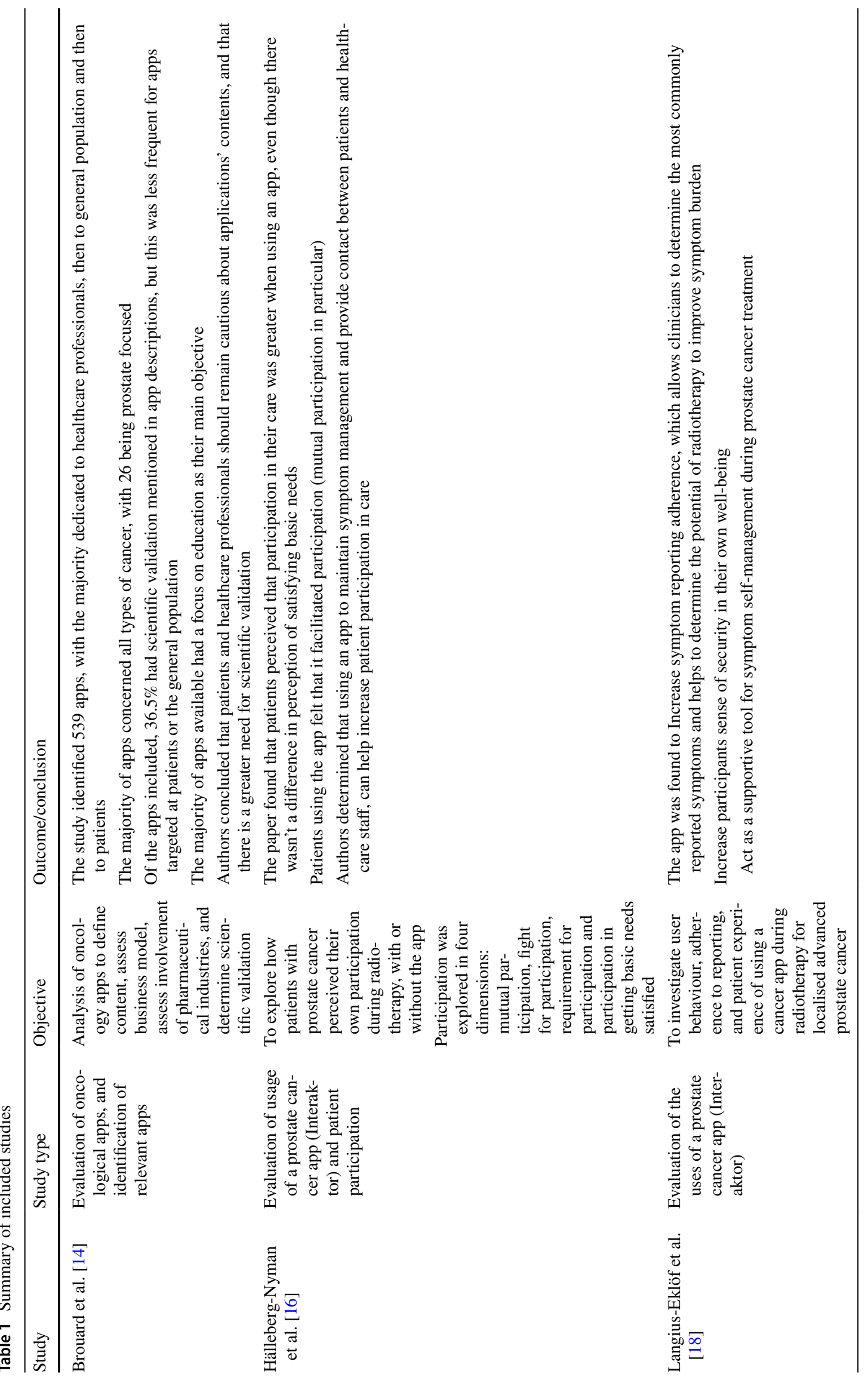




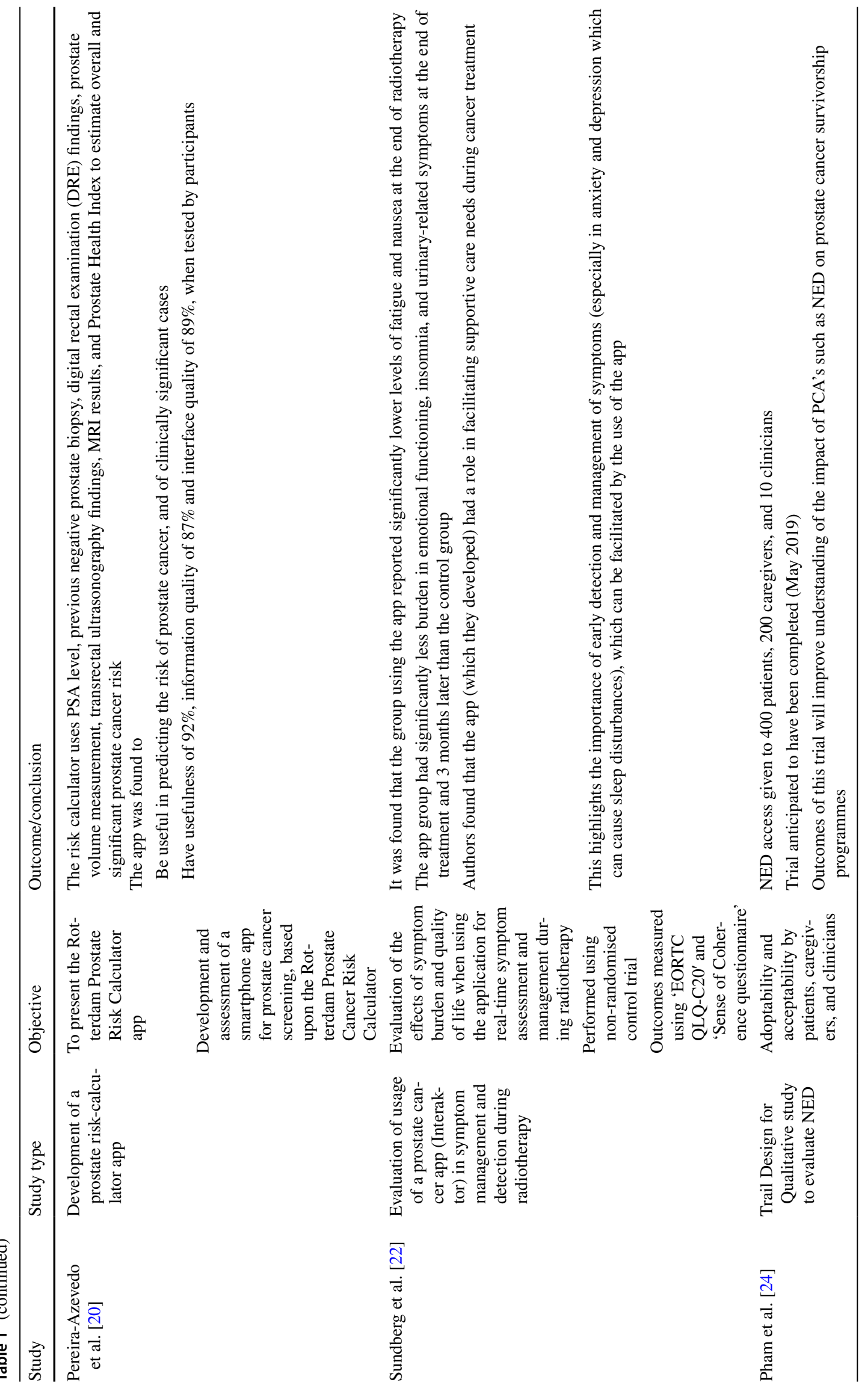




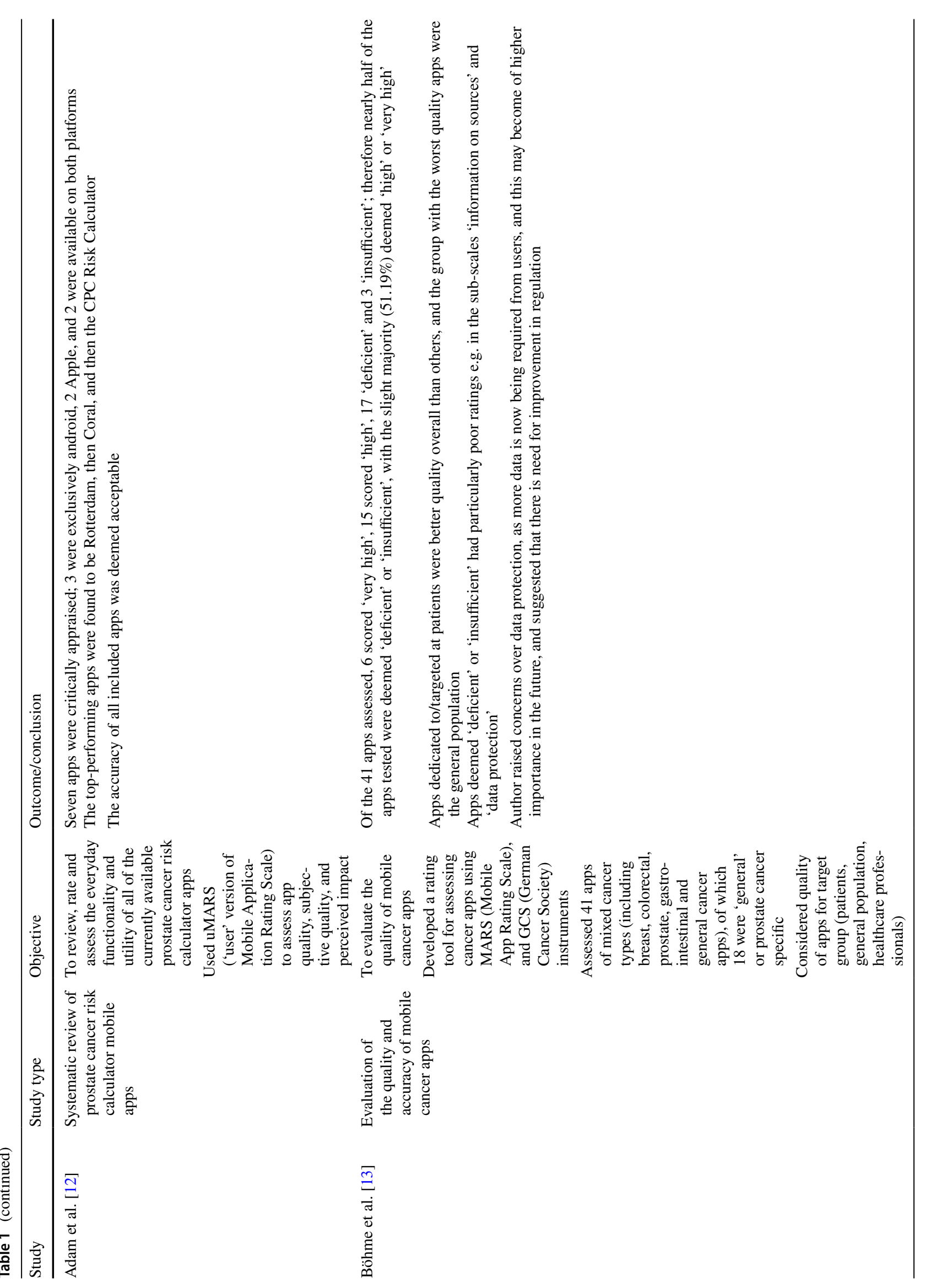




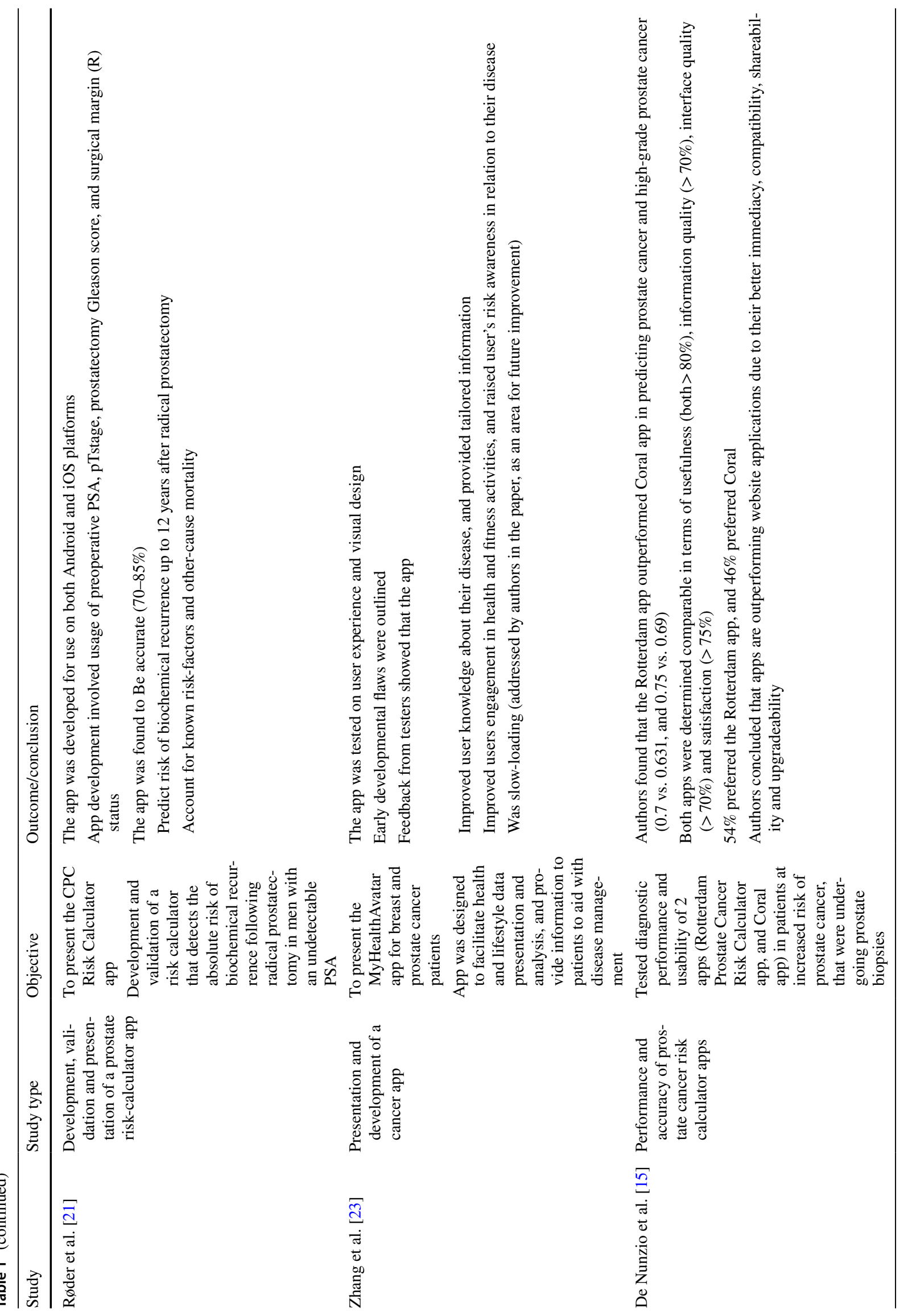




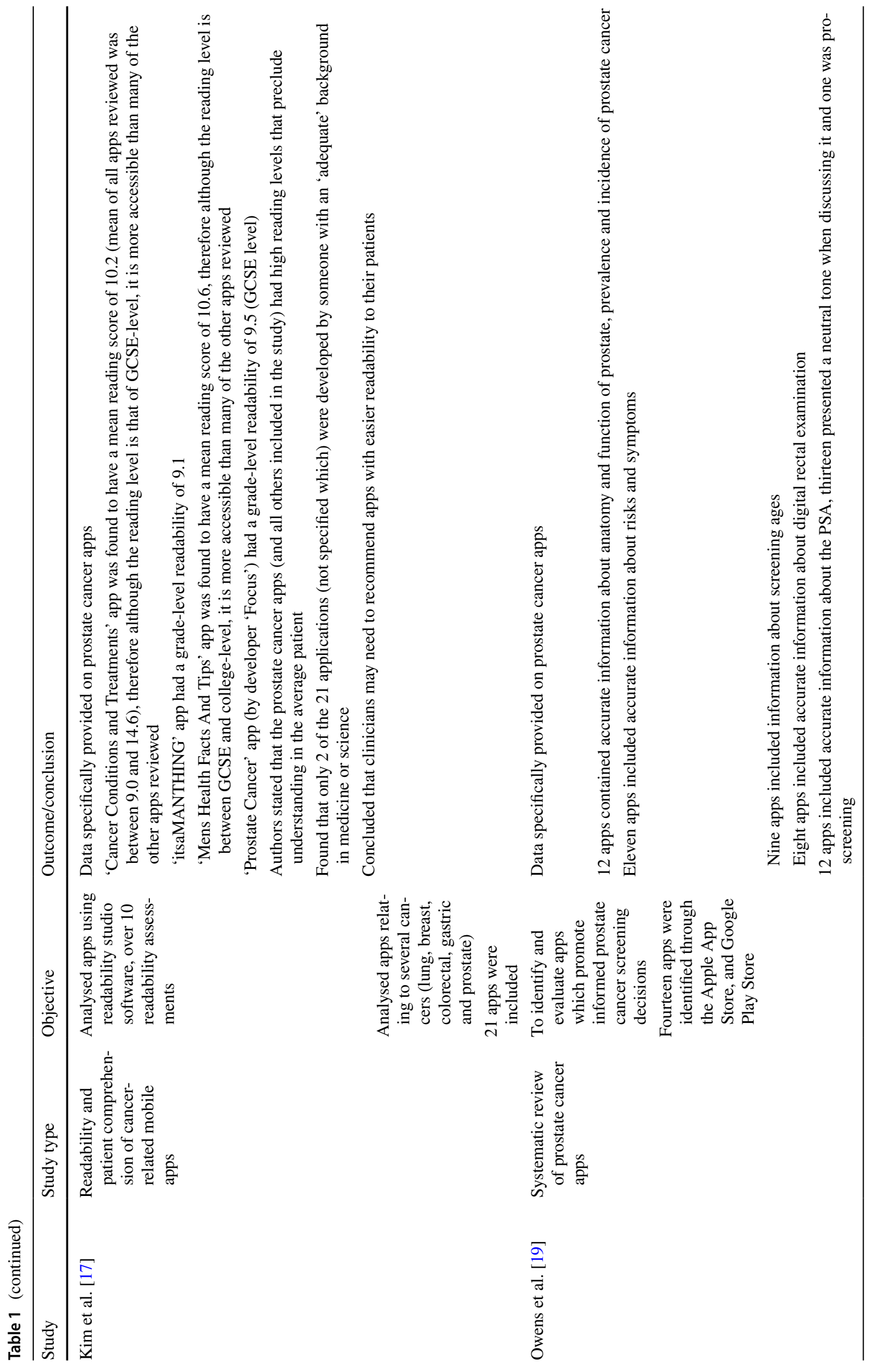




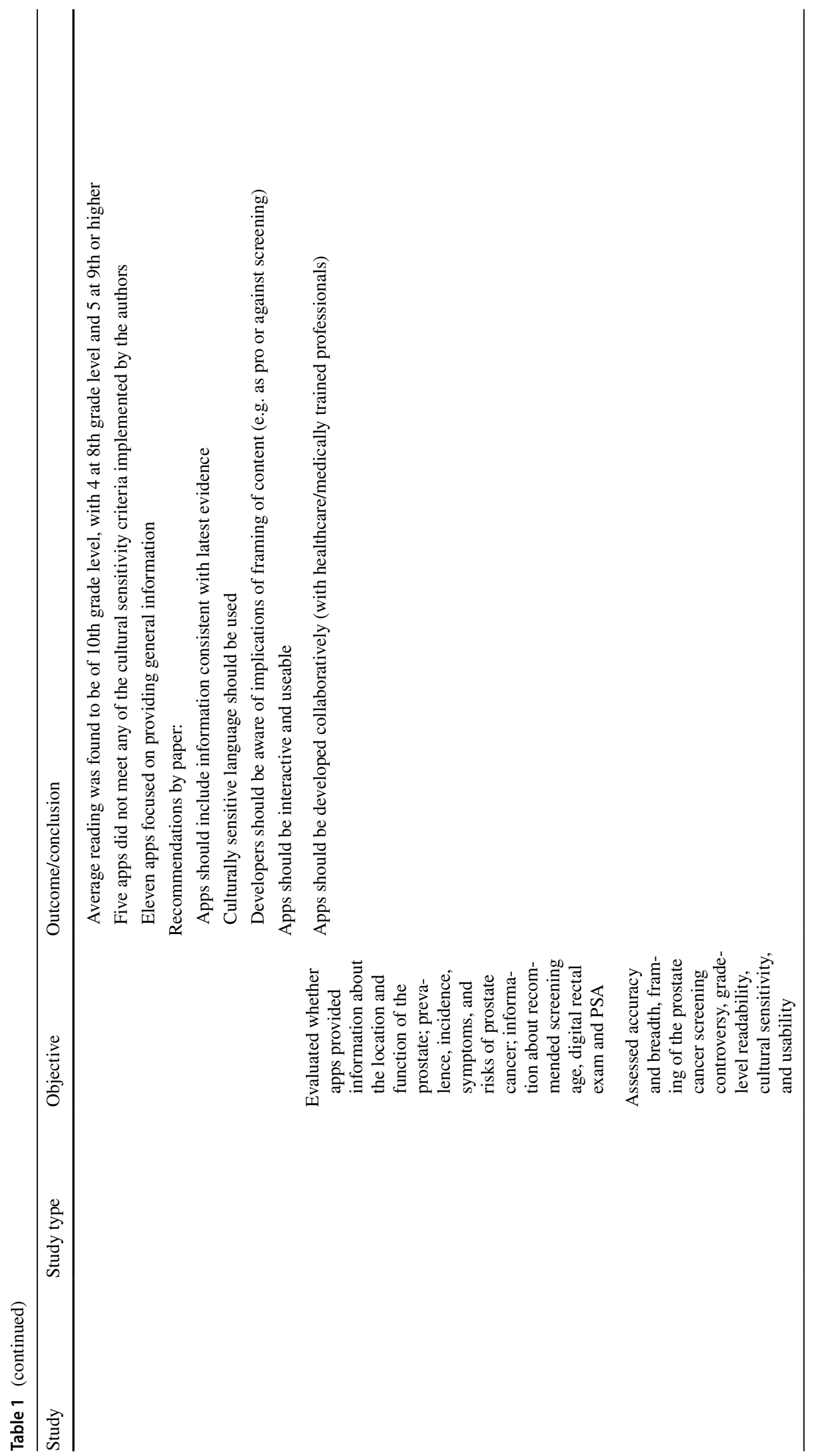


Apple Store and Google Play Store. The study identified 12 articles from 3 PCAs (Mens Health Facts and Tips, ProstAid, Prostate Cancer) for evaluation. The study reported that PCAs available on Apple Store and Google Play Store had average reading grades of 10.6 and 9.4 respectively [17]. Owens et al. identified 14 PCAs. 10 PCAs had adequate material for readability evaluation with the average reading to be at 10th grade level [19]. The study concluded that the apps included in the study were of high reading levels that were greater than that of the average patient, which might prevent patients from understanding the information they contain.

\section{Studies related to 'quality and accuracy of apps'}

Owens et al. [19] also explored the quality of content provided by 14 PCAs. This was based upon accuracy, breadth, tone/framing and cultural sensitivity of the app's content. Best Prostate Cancer Treatment, Oncotip, and Prostate Cancer by Magna Health Solutions were the 3 PCAs that had most extensive detail on prostate cancer covered. Authors found that 13 of the 14 apps studied had a neutral tone with regards to PSA testing. Oncotip was the only PCA that was pro-screening. Overall, the majority of apps tested provided an accurate and wide range of information and were of good quality. The overall rating of the " 14 PCAs for cultural sensitivity for African Americans was low. Procee had the best rating for cultural sensitivity [19].

Bohme et al. [13] evaluated the quality of apps, using the Mobile Application Rating Scale (MARS) and German Cancer Society (GCS) instruments to determine the quality of information contained within the apps for breast, prostate and colorectal cancer. The tools had 3 domains (engagement, aesthetics and information) and 22 aspects were evaluated. 24 apps in the study were PCAs. Of all apps included in the study, around $48.78 \%$ were considered deficient or insufficient [13]. The authors reported the overall quality of apps which were targeted at patients were of better quality than those targeted at either healthcare professionals or the general population. The study highlighted deficient or insufficient scores for data protection.

\section{Studies related to 'app usage'}

Three papers examined the usage of PCAs [14, 16, 22]. Hälleberg-Nyman et al. [16] and Sundberg et al. [22] both assessed the app 'Interaktor' and determined it to be a useful tool. Sundberg et al., found that when using the app for the real-time assessment of symptoms in prostate cancer patients undergoing radiotherapy, the control group (who did not use the app) displayed significantly worse emotional functioning at the end of radiotherapy when compared to the intervention group. Authors posited that this highlights the importance of the early detection and management of symptoms - something which apps can facilitate [22]. Furthermore, Hälleberg-Nyman et al. [16] found that patients using the app had a greater perceived participation in their care, which may be important in doctor-patient relationships and patient outcomes.

Brouard et al. [14] found that the majority of apps available had a focus on education as their main objective. They also found that the apps included in their study which were aimed at patients or the general population had less scientific validation than those targeted towards healthcare professionals [14].

\section{Studies related to 'risk calculators'}

Two papers solely explored risk-calculator applications and their accuracy $[12,15]$. De Nunzio et al., compared the performance of Rotterdam [27] and Coral [28] in 1682 patients undergoing prostate biopsies for suspected prostate cancer. Rotterdam was significantly better than the Coral at predicting overall (AUC: 0.70 versus $0.631, p=0.001$ ) and highgrade prostate cancer $(0.75$ versus $0.69, p=0.001)$. Both apps were accurate and comparable in terms of usefulness (both $>80 \%$ ), information quality ( $>70 \%$ ), interface quality ( $>70 \%)$ and satisfaction (>75\%) [28]. However, 54\% of participants preferred Rotterdam overall. Rotterdam also was deemed the best by Adam et al., who critically appraised 7 applications across both Android and iOS platforms [12, 15]. They found that the top-performing apps when using the uMARS scale (user version of the MARS scale) to assess quality, were Rotterdam, then Coral, and then the CPC Risk Calculator, although accuracy of all included apps was deemed acceptable [12].

\section{Individual applications includes studies evaluated by the authors}

Of the 12 papers included in the study, 44 apps were identified for which we collected data. Due to the rapidly developing nature of applications, at the time of the study 11 apps were no longer available to evaluate. The remaining 33 apps were mainly free, with only 2 requiring a subscription. As found by authors of the papers included in this review, the majority (59\%) of the apps had an educational focus, with other objectives being risk assessment, support, or targeted towards clinicians for information or decision making. Interestingly only 5 of the apps had been updated within the last year, which may suggest that information within the other apps may not entirely up to date. The full analysis and breakdown of these apps and their content can be seen in Table 2 . 
2422

World Journal of Urology (2020) 38:2411-2431

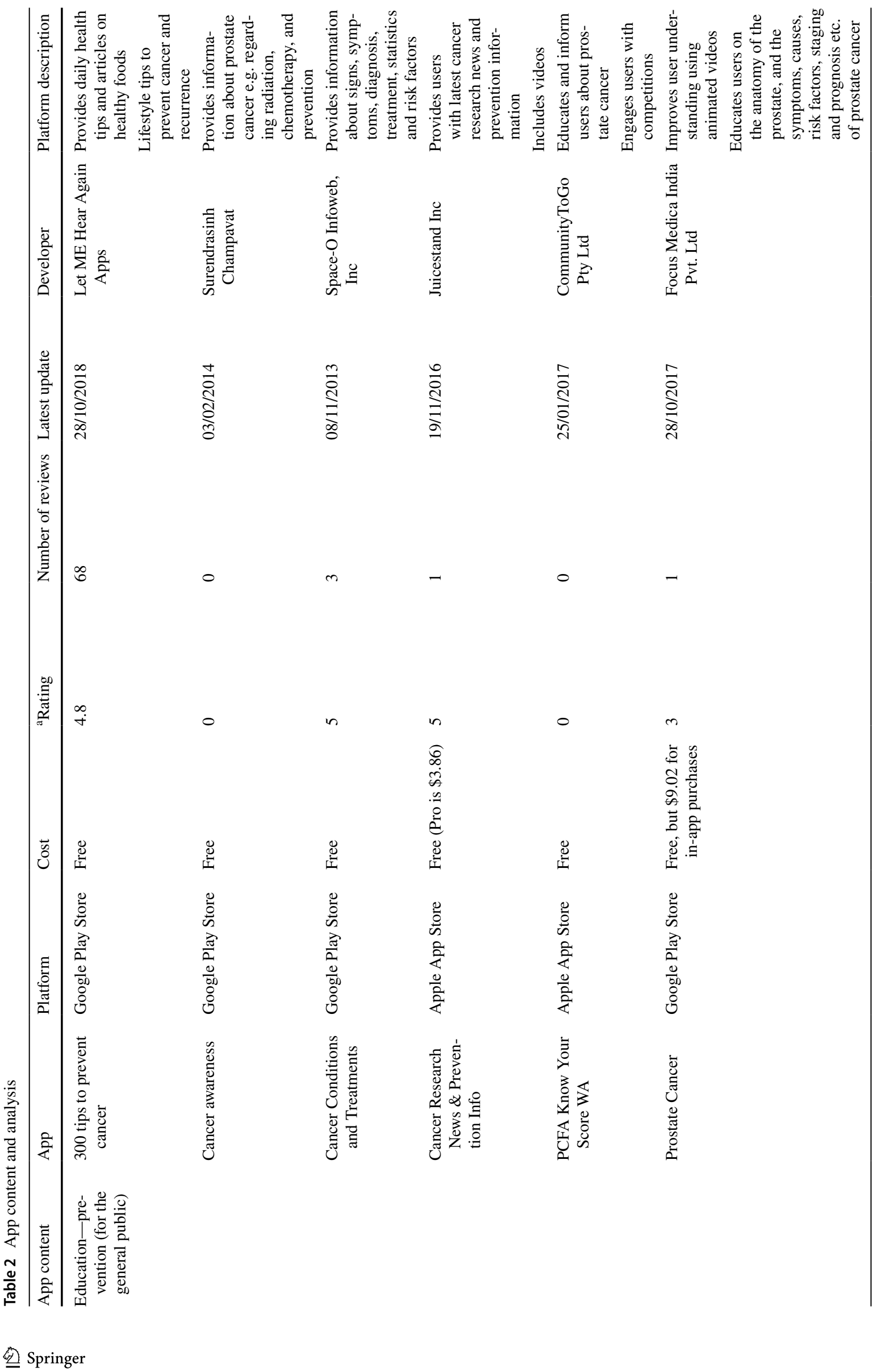




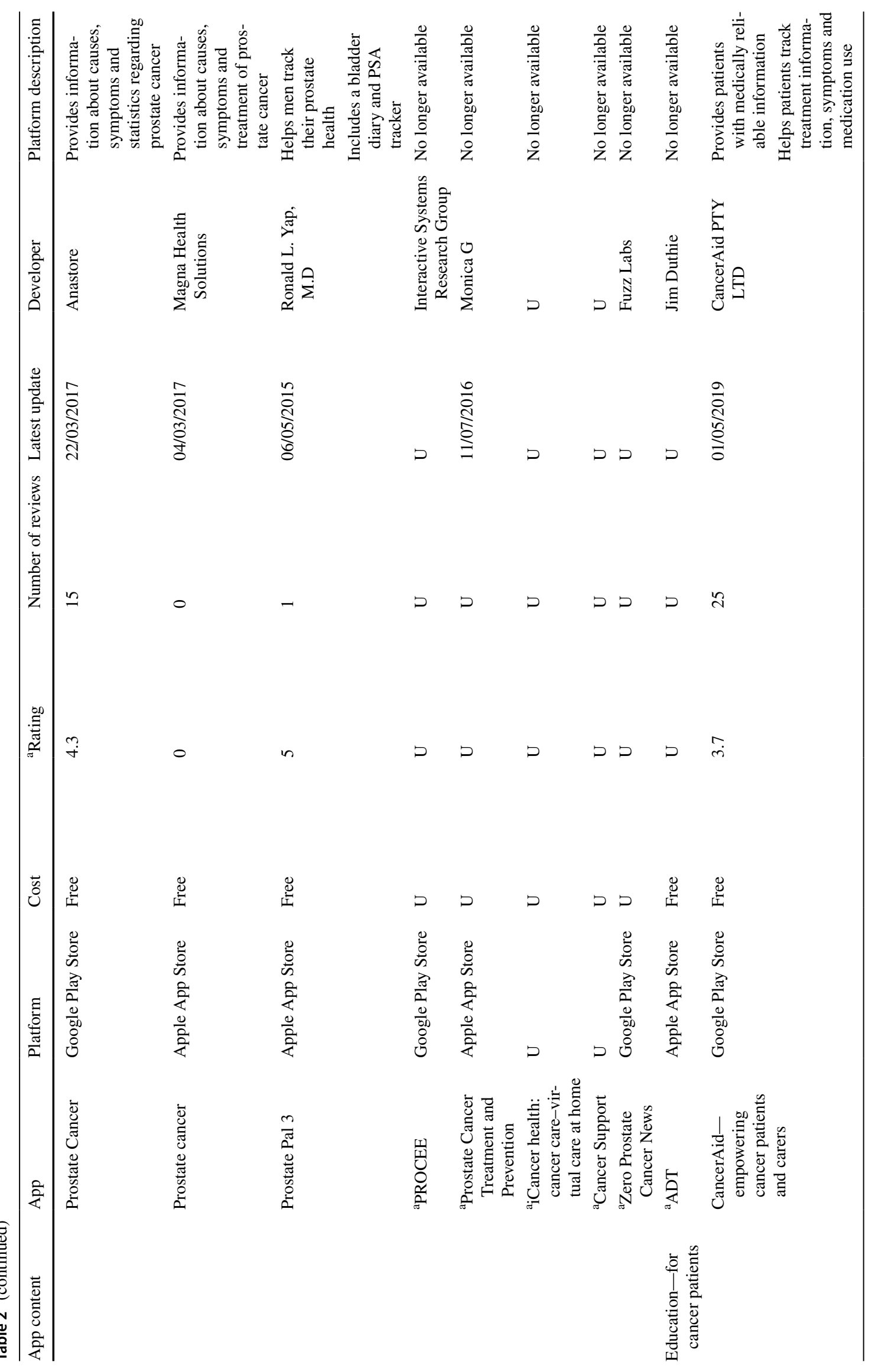




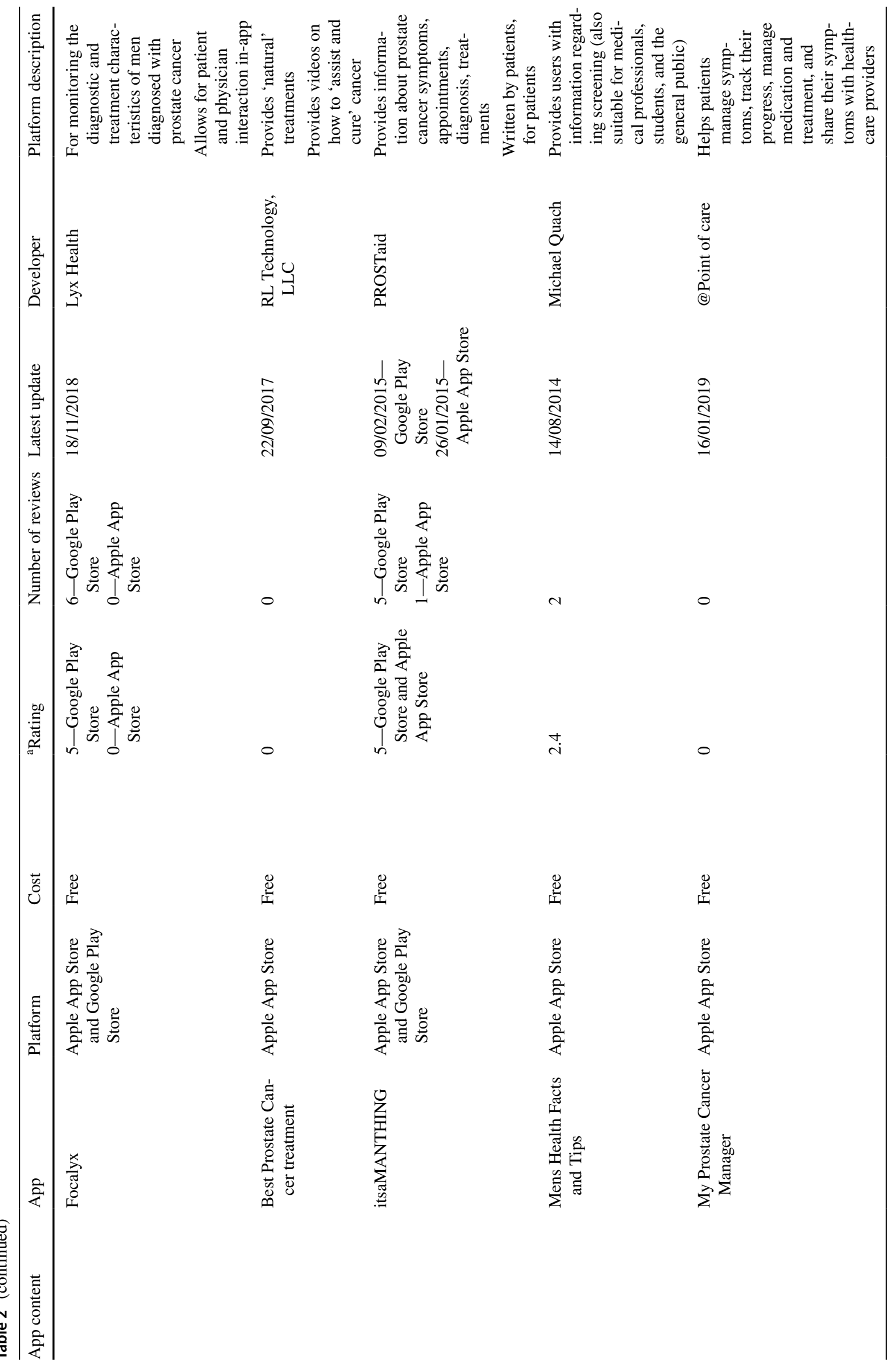




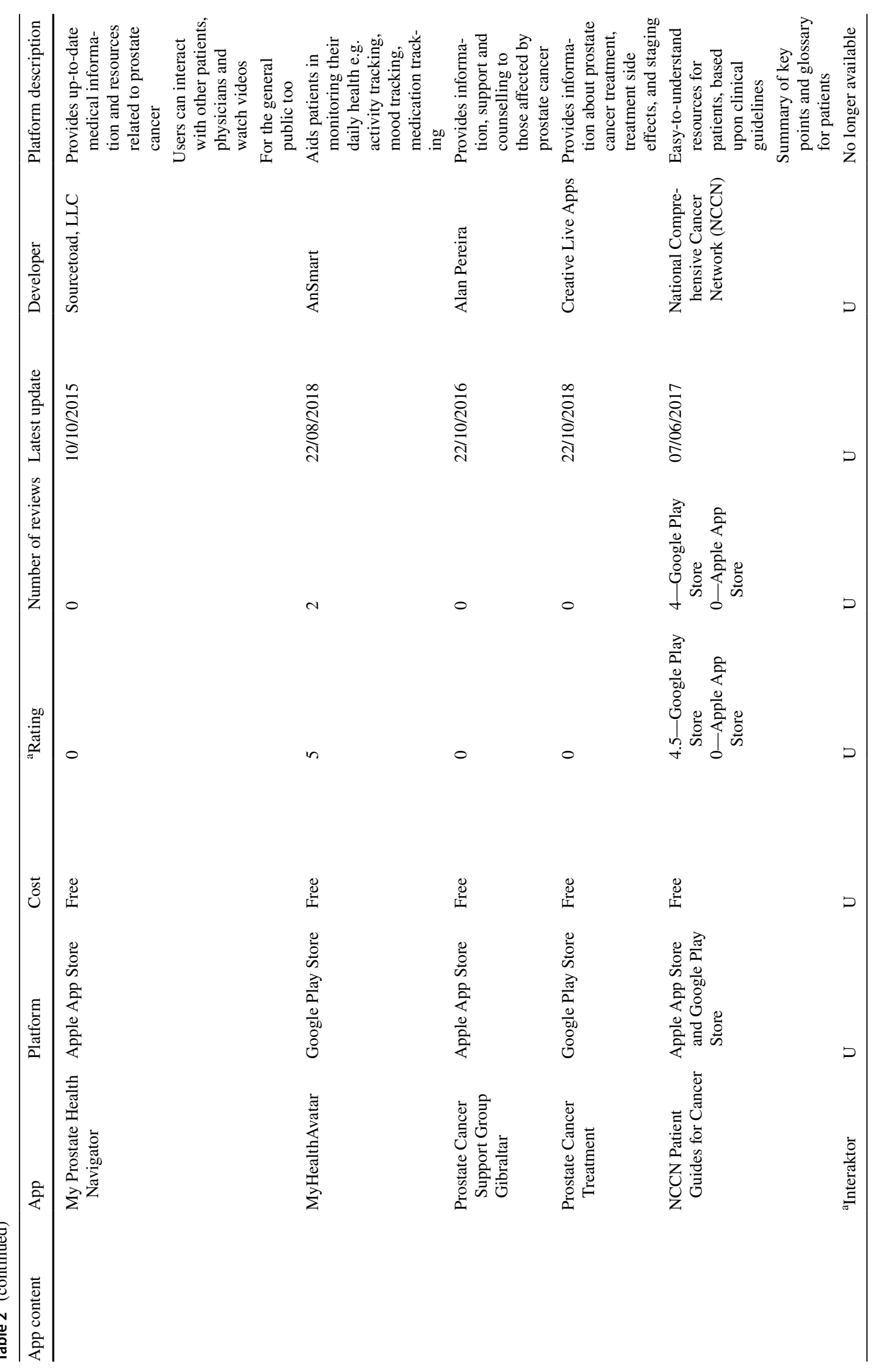




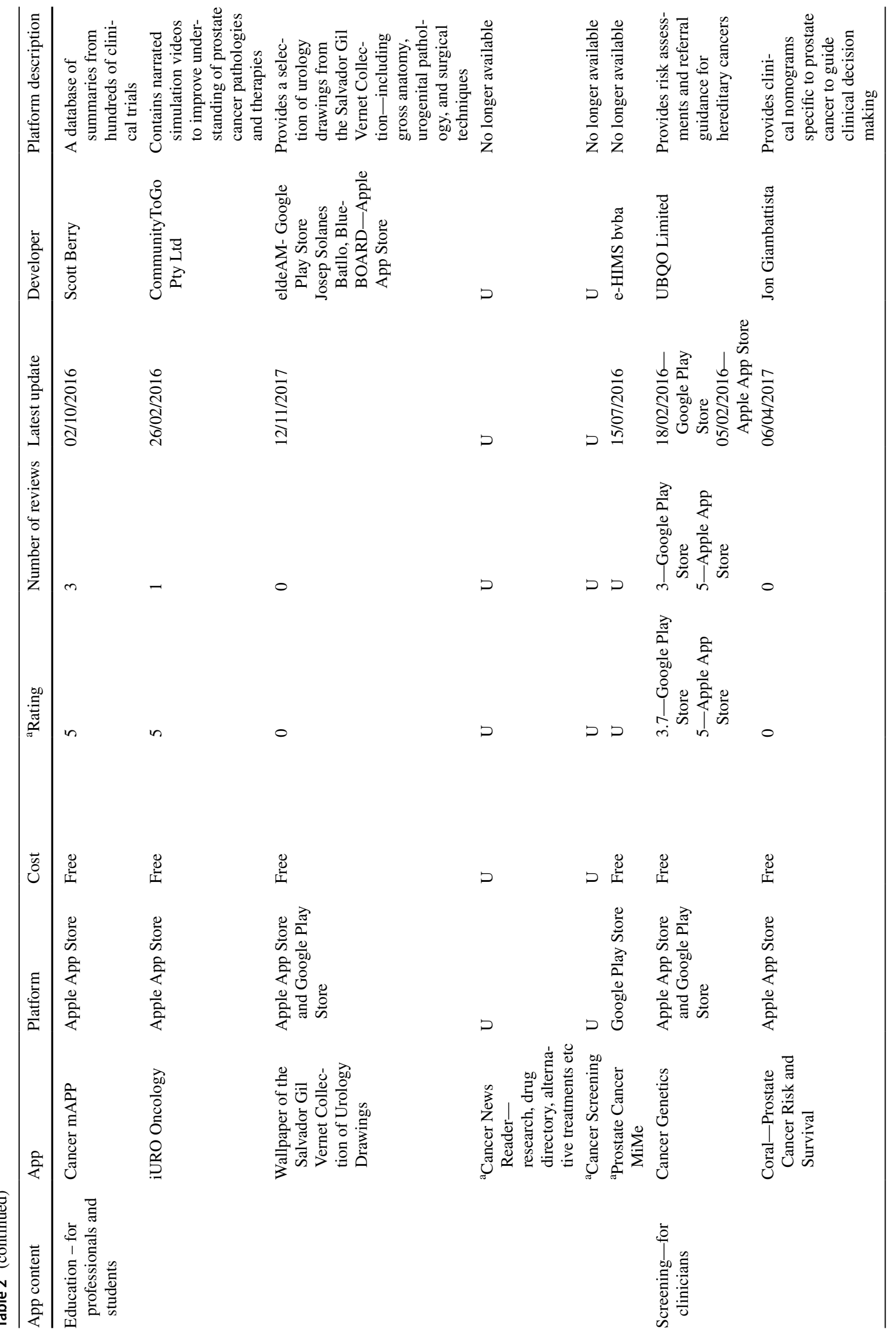




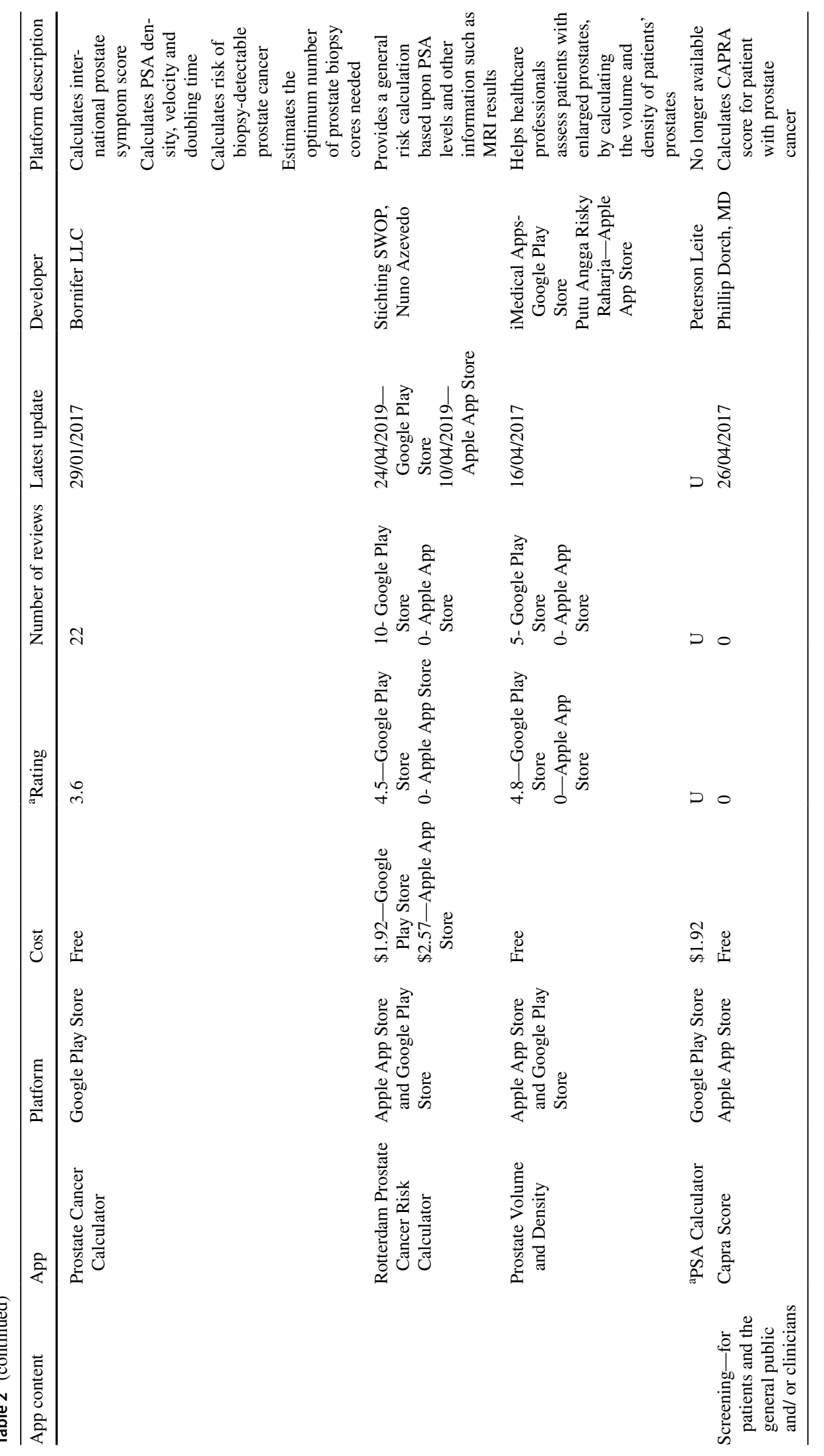


2428

World Journal of Urology (2020) 38:2411-2431

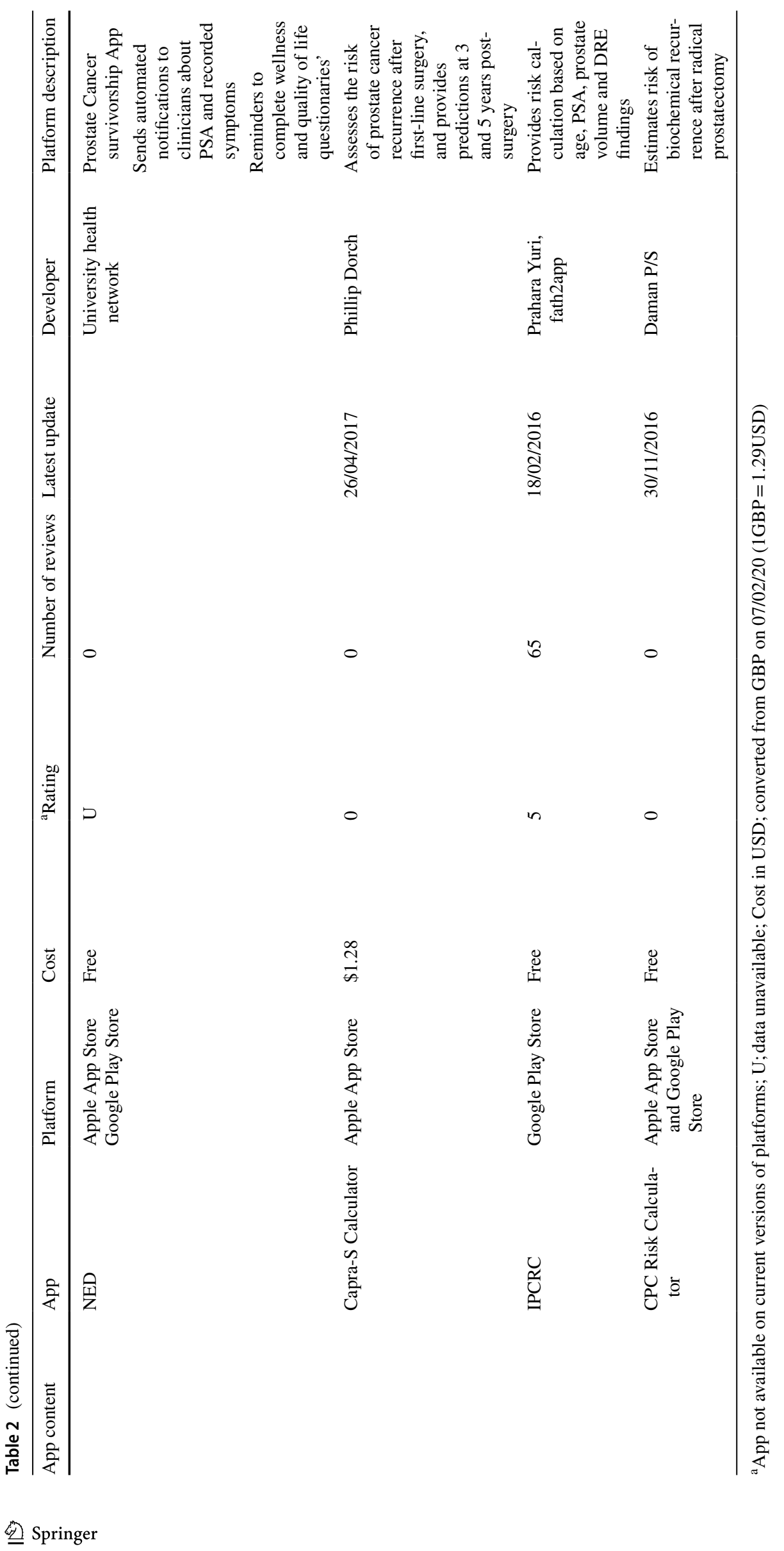




\section{Discussion}

This systematic review has identified 44 PCAs targeting the general population, patients and clinicians, with a majority (33 of 44) of them focusing on education. It is the authors view that existing PCA's are currently in its infancy and do require further development before widespread integration into existing clinical practise. The apps covered topics such as lifestyle changes, and information on prostate cancer including treatment options, PSA screening, symptomatology, diagnostics, statistics, research and prostate anatomy. Three prostate cancer risk calculators (Rotterdam, CORAL and CPC) were identified which provided estimates on prostate cancer diagnosis and biochemical recurrence following radical prostatectomy. Rotterdam was the best performer amongst the 3 risk calculators. Most PCAs were rated to have a high standard of readability, raising concerns that a proportion of the patient population may not be able to adequately comprehend the available information in them. Additionally, one study reported deficient or insufficient quality for data protection for cancers apps [13]. The gross majority ( 36 of 43) of the PCAs haven't been updated in the last year and, therefore, there is doubt if the existing data in these apps is current.

PCAs have the potential to have a number of roles in the contemporary management of prostate cancer. Healthcare organisations world-wide have adopted the principles of shared care decision making (SDM) between a healthcare professional and patients $[29,30]$. PCAs, in addition to existing Decision Aids (DA) can be useful adjuncts to clinical counselling, facilitating well-informed clinical decision making, improve clinician-patient communication and as a consequence leading to a favourable patient experience [29]. PCAs such as Interaktor and MyHealthAvatar (MHA) are such PCAs that have been developed as supportive aids that compliment clinical consults. Hälleberg-Nyman et al. [16] in qualitative study reported patient-reported satisfaction scores to be better in patients receiving radiotherapy, when clinical consults were supplemented with the interactive app, Interaktor, corroborating the aforementioned view. In prostate cancer, SDM with DAs is particularly pertinent, due to controversies in areas such as prostate cancer screening and the availability of plethora of therapeutic options [31]. In this review, a number of PCAs addressed the subject of PSA screening and reassuringly most PCAs had a neutral tone for PSA screening. PCAs can be useful adjuncts to clinical consults in this context, conforming to the principles of informed patient choice and avoiding decision regret.

Prostate cancer diagnostics has seen significant evolution in recent years with strategies such as pre-biopsy multi-parametric MRIs [32]. This trend is likely to continue with the pursuit for biomarker technologies in prostate cancer [32].
Assimilation and presentation of ever-growing data from existing and novel diagnostic tools, in short clinical consults can be challenging. PCAs such as Rotterdam and CORAL integrate data from diagnostic tools and demographics, subsequently presenting an estimated risk of prostate cancer $[27,28]$. PCAs such as Rotterdam and CORAL are hence invaluable aids to clinicians, allowing for seamless, efficient and accurate patient counselling. However, it is important to note that whilst Rotterdam does include MRI results as a criterion, CORAL does not, which may affect the accuracy of the result. Similarly, biochemical recurrence predictions following curative local treatments can be challenging and CPC calculators are therefore useful tools for clinicians [26]. Innovative Prostate cancer survivorship programmes will be required to manage an increasing population of prostate cancer survivors. Chu et al. [33] in a retrospective review reported over $95 \%$ patient satisfaction rates and individual patient savings of 193 US dollars with telemedicine delivered care. PCAs such as NED lineate well with prostate survivorship programmes and can be employed for post-treatment surveillance without the need for periodic attendance at hospital. This has benefits to patients living in remote locations with poor health care accessibility and also cost-saving benefits to healthcare organisations and individual patients.

Predictive analytics is increasing being adopted in healthcare to improve operational efficiency and disease management [34]. Studying behavioural and lifestyle patterns across a wide range of demographics can facilitate the identification of causal relationships. Medical apps are a useful information communication technology for large volume real-world data collection mitigating some of the challenges of traditional data collection. MyHealthAvatar (MHA) is PCA that has the ability to collate demographic, behavioural, lifestyle, and medical data for prostate cancer patients [23, 25]. These allow for analysing data in multiple clinical scenarios and can, therefore, lead to the creation of various virtual patient populations. These provide invaluable data to healthcare providers which may contribute to future stratified individualised care [35].

Despite the potential benefits of medical health apps in general and PCAs specifically, the potential for harm is real. PCAs must be accurate, easily comprehendible, un-biased and regularly updated. This review suggests that PCAs do not consistently fulfil all these pre-requisites. Brouard et al. [14] reported a majority of medical apps targeted at patients and the general population haven't had scientific validation. Misinformation can lead to anxiety, over-diagnosis and overtreatment. It is therefore vital these apps are appropriately governed by stringent regulation to ensure patient safety. In Europe and the United Kingdom, current guidelines recommend only app with a CE marking are approved for clinical use [36]. Local institutions would be advised to have agreed on protocol of PCAs usage in clinical practise [36]. Bohme 
et al. [13] reported most cancer apps to deficient or insufficient in data protection and, therefore, caution must be exercised before patient sensitive information is added to these apps. Furthermore, healthcare professionals must be provided with formal education on the potential harms of modern day medical apps so as to ensure responsible usage.

Limitations of our study included the exclusion of grey literature, and papers not written in English. Although there are other sources of social media such as twitter, YouTube and google search engines, however in this paper we focussed on the telephone-based apps only. Due to the constant changes in the nature of apps, older software was not always updated and occasionally removed in time, hence our inability to find some of the apps mentioned in the papers analysed.

\section{Conclusion}

There are a wide variety of PCAs available targeting the general population, patients and clinicians, with a majority of them focusing on education. The apps covered topics such as lifestyle changes, and information on prostate cancer including treatment options, PSA screening, symptomatology, diagnostics, statistics, research and prostate anatomy. A number of PCAs haven't undergone scientific validation. There are concerns with data protection, high readability standards and lack of information update in current PCAs. There must be increased awareness among patients and clinicians about existing PCAs and their limitation so as to ensure safe and responsible usage. It is the authors view that existing PCAs are currently in its infancy and do require further development before widespread integration into existing clinical practise. If developed appropriately with responsible governance, they do have the potential to play important roles in modern day prostate cancer management.

Open Access This article is licensed under a Creative Commons Attribution 4.0 International License, which permits use, sharing, adaptation, distribution and reproduction in any medium or format, as long as you give appropriate credit to the original author(s) and the source, provide a link to the Creative Commons licence, and indicate if changes were made. The images or other third party material in this article are included in the article's Creative Commons licence, unless indicated otherwise in a credit line to the material. If material is not included in the article's Creative Commons licence and your intended use is not permitted by statutory regulation or exceeds the permitted use, you will need to obtain permission directly from the copyright holder. To view a copy of this licence, visit http://creativecommons.org/licenses/by/4.0/.

\section{References}

1. Aceto G, Persico V, Pescapé A (2018) The role of Information and communication technologies in healthcare: taxonomies, perspectives, and challenges. J Netw Comput Appl 107:125-154
2. Maurer T, Eiber M (2019) Practice changing for prostate cancer: a vision of the future. Nat Rev Urol 16(2):71-72

3. Techopedia (2019) Mobile application. https://www.techopedia .com/definition/2953/mobile-application-mobile-app. Accessed 6 Aug 2019

4. Jamnadass E, Aboumarzouk O, Kallidonis P, Emiliani E, Tailly T, Hruby $S$ et al (2018) The role of social media and internet search engines in information provision and dissemination to patients with kidney stone disease: a systematic review from european association of urologists young academic urologists. J Endourol 32(8):673-684

5. Iqbal M (2019) App download and usage statistics (2019). https ://www.businessofapps.com/data/app-statistics/. Accessed 6 Aug 2019

6. Kemp S (2019) Digital 2019: global internet use accelerates. https://wearesocial.com/blog/2019/01/digital-2019-global-inter net-use-accelerates. Accessed 6 Aug 2019

7. Wurmser Y (2019) Mobile time spent 2018-will smartphones remain ascendant? https://www.emarketer.com/content/mobil e-time-spent-2018. Accessed 6 Aug 2019

8. Colwyn S (2019) New consumer media consumption research. https://www.smartinsights.com/marketplace-analysis/customeranalysis/consumer-media-device-use/. Accessed 6 Aug 2019

9. Redbytes (2019) Growing prevalence of healthcare mobile apps in the UK. https://www.redbytes.co.uk/mobile-health-app-usage -statistics/. Accessed 6 Aug 2019

10. Higgins JP, Green S (2008) Cochrane handbook for systematic reviews of interventions. Wiley, Chichester

11. Moher D, Liberati A, Tetzlaff J et al (2009) Preferred reporting items for systematic reviews and meta-analyses: the PRISMA statement. PLoS Med 6(7):e1000097

12. Adam A, Hellig JC, Perera M, Bolton D, Lawrentschuk N (2018) 'Prostate Cancer Risk Calculator' mobile applications (Apps): a systematic review and scoring using the validated user version of the Mobile Application Rating Scale (uMARS). World J Urol 36(4):565-573

13. Bohme C, von Osthoff MB, Frey K, Hubner J (2018) Qualitative evaluation of mobile cancer apps with particular attention to the target group, content, and advertising. J Cancer Res Clin Oncol 144(1):173-181

14. Brouard B, Bardo P, Bonnet C, Mounier N, Vignot M, Vignot S (2016) Mobile applications in oncology: is it possible for patients and healthcare professionals to easily identify relevant tools? Ann Med 48(7):509-515

15. De Nunzio C, Lombardo R, Tema G, Cancrini F, Russo GI, Chacon R et al (2019) Mobile phone apps for the prediction of prostate cancer: External validation of the Coral and Rotterdam apps. Eur J Surg Oncol 45(3):471-476

16. Halleberg Nyman M, Frank C, Langius-Eklof A, Blomberg K, Sundberg K, Wengstrom Y (2017) Patients' perspective on participation in care with or without the support of a smartphone app during radiotherapy for prostate cancer: qualitative study. JMIR Mhealth Uhealth 5(7):e107

17. Kim C, Prabhu AV, Hansberry DR, Agarwal N, Heron DE, Beriwal S (2019) Digital era of mobile communications and smartphones: a novel analysis of patient comprehension of cancerrelated information available through mobile applications. Cancer Invest 37(3):127-133

18. Langius-Eklof A, Christiansen M, Lindstrom V, Blomberg K, Halleberg Nyman M, Wengstrom Y et al (2017) Adherence to report and patient perception of an interactive app for managing symptoms during radiotherapy for prostate cancer: descriptive study of logged and interview data. JMIR Cancer 3(2):e18

19. Owens OL, Beer JM, Reyes LI, Thomas TL (2019) Systematic review of commercially available mobile phone 
applications for prostate cancer education. Am J Mens Health 13(1):1557988318816912

20. Pereira-Azevedo N, Osorio L, Fraga A, Roobol MJ (2017) Rotterdam prostate cancer risk calculator: development and usability testing of the mobile phone app. JMIR Cancer 3(1):e1

21. Roder MA, Berg KD, Loft MD, Thomsen FB, Ferrari M, Kurbegovic $\mathrm{S}$ et al (2018) The CPC risk calculator: a new app to predict prostate-specific antigen recurrence during follow-up after radical prostatectomy. Eur Urol Focus 4(3):360-368

22. Sundberg K, Wengstrom Y, Blomberg K, Halleberg-Nyman M, Frank C, Langius-Eklof A (2017) Early detection and management of symptoms using an interactive smartphone application (Interaktor) during radiotherapy for prostate cancer. Support Care Cancer 25(7):2195-2204

23. Zhang X, Deng Z, Parvinzamir F, Dong F (2018) MyHealthAvatar lifestyle management support for cancer patients. Ecancermedicalscience 12:849

24. Pham Q, Cafazzo JA, Feifer A (2017) Adoption, acceptability, and effectiveness of a mobile health app for personalized prostate cancer survivorship care: protocol for a realist case study of the ned app. JMIR Res Protoc 6(10):e197

25. AnSmart (2018) MyHealthAvatar. Accessed 22 Aug 2018

26. Daman PS (2016) CPC Risk Calculator. Accessed 30 Nov 2016

27. Stichting SWOP, Azevedo N (2019) Rotterdam Prostate cancer risk calculator In: 24/04/2019 on Android, 10/04/2019 on iOS

28. Giambattista J (2017) Coral-prostate cancer risk and survival. Accessed 6 Apr 2017

29. Stacey D, Légaré F, Lewis K, Barry MJ, Bennett CL, Eden KB et al (2017) Decision aids for people facing health treatment or screening decisions. Cochrane Database of Syst Rev 2017:4
30. (2019) https://www.england.nhs.uk/rightcare/useful-links/share d-decision-making/. Accessed 26 Oct 2019

31. Riikonen JM, Guyatt GH, Kilpelinen TP, Craigie S, Agarwal A, Agoritsas T et al (2019) Decision aids for prostate cancer screening choice: a systematic review and meta-analysis. JAMA Internal Med 179(8):1072-1082

32. Logothetis CJ, Aparicio A, Koinis F, Corn PG, Efstathiou E (2019) Prostate cancer: quo vadis? Eur Urol 76(6):709-711. https ://doi.org/10.1016/j.eururo.2019.06.031

33. Chu S, Boxer R, Madison P, Kleinman L, Skolarus T, Altman L et al (2015) Veterans affairs telemedicine: bringing urologic care to remote clinics. Urology 86(2):255-260

34. Chen J, Remulla D, Nguyen JH, Aastha D, Liu Y, Dasgupta P et al (2019) Current status of artificial intelligence applications in urology and their potential to influence clinical practice. BJU Int 124(4):567-577

35. Spanakis EG, Yang, P, Deng Z, Sakkalis V, Kafetzopoulos D., Marias, K, Tsiknakis MN, Dong F (2014) MyHealthAvatar: personalized and empowerment health services through Internet of Things technologies. In: 4th international conference on wireless mobile communication and healthcare

36. https://www.gov.uk/government/publications/medical-devicessoftware-applications-apps. Accessed 26 Oct 2019

Publisher's Note Springer Nature remains neutral with regard to jurisdictional claims in published maps and institutional affiliations. 